Original Research Paper

\title{
An Efficient Multimodal Biometric Authentication Integrating Fingerprint and Face Features
}

\author{
Shohel Sayeed, Ilham Nasir and Thian Song Ong \\ Faculty of Information Science and Technology, \\ Multimedia University, Jalan Ayer Keroh Lama, 75450 Melaka, Malaysia
}

Article history

Received: 31-08-2016

Revised: 29-09-2016

Accepted: 14-11-2016

Corresponding Author:

Shohel Sayeed

Faculty of Information Science and Technology, Faculty of

Information Science and

Technology, Multimedia

University, Jalan Ayer Keroh

Lama, 75450 Melaka, Malaysia

Email: shohel.sayeed@mmu.edu.my

\begin{abstract}
Biometrics authentication has been used in many applications such as e-commerce, access control etc. In this research, multimodal biometric authentication in light of score level combination of fingerprint and face is proposed. Face and fingerprint is two of the most popular biometric traits and can complement each other for more reliable user authentication. To verify the effectiveness of the proposed method, FERET, SDUMLA-HMT and FVC2002 databases are used in this research. Furthermore, experimental results showed that the proposed fusion based method for fingerprint and face recognition can improve the performance in terms of Equal Error Rate (EER). Eventually, the experimental result shows that our proposed fusion based technique appears to be promising with $7.01 \sim 9.13 \%$ of EER based on the different datasets.
\end{abstract}

Keywords: Biometrics, Multimodal, Fingerprint, Face, Features, Fusion

\section{Introduction}

In this era, there are various situations that people need to authenticate themselves. Authentication is a process to determine that someone is really the person that he claimed to be or not. Generally, there are three types of authentication which are something you know, something you have and something you are. Password is an example for something you know. Password is the most common technique used by people to authenticate themselves. However, password needs a complex combination to make it secure and it is not easy to remember a complex combination. In addition, the example of something you have is token. Token will generate a random number then people need to input that random number into the system. Next, a system will verify that random number. However, it is not convinient to carry token anywhere as it might be lost or stolen. The last authentication type is something you are. People can authenticate themselves without remembering the complex combination or carry any devices. People only need their own traits to authenticate themselves, for example, their eyes, retina, hand, fingerprint, etc. This authentication method is called biometrics. Biometrics uses physiological or behavioural human traits for authentication purposes. The physiological traits include face, fingerprint, palm, retina and etc and the behavioral traits include gait, speech, signature and etc. Based on the number of traits, biometrics can be categorized as unimodal and multimodal. Unimodal biometrics is a biometric system that use single trait for authentication. This type of biometrics has some limitations such as noisy sensor data, spoof attack, non-universality and unacceptable error rate.

On the other hand, multimodal biometrics is a biometric system that use more than one characteristic of recognition. Multimodal biometrics can overcome limitation of unimodal biometrics because it has multipme information from different sources (Sarhan et al., 2016). Furthermore, multimodal biometrics has some advantages like more robust to noise, improve the accuracy, more secure and can solve the non-universality problem.

Thus, the development of multimodal biometric is fast and there are many kinds of combinations of biometrics, such as fingerprint and finger vein, face and iris, face and gait, iris and retina.

In this study, multimodal biometric authentication that based on face and fingerprint is proposed. The fusion between face and fingerprint is proposed because both of them are the most popular biometric traits and the device for capturing face and fingerprint images is affordable.

\section{Fusion Levels of Multimodal Biometrics}

In multimodal biometrics, there are various level of fusion possibility, such as: 
- Data level: Fused the original biometrics data, for example: Combining face and fingerprint image

- Feature level: Combining the extracted biometric features with another biometric features

- Score level: Matching score of different biometric systems are fused together and produce a single score

- Decision level: Combining the decision that has been made from each of biometric system

Each of fusion level has its own strengths and weaknesses. Table 1 shows some advantages and disadvantages of each of fusion level.

It is believed that fusion on the earlier level is better. Feature level fusion is expected to provide a better result compare with score level or decision level because feature contains more information. Notwithstanding, the combination at feature level is troublesome in light of the fact that list of the feature set of various modalities is not compatible with each other. Thus, fusion in score level is better because it is not hard to get and fusion the matching score from different modalities (Ross and Jain, 2003; 2004).

\section{Proposed Method for Fingerprint and Face Recognition}

\section{Fingerprint Recognition}

In this study, the minutiae-based technique is chosen for fingerprint recognition because of its simplicity and robustness. There are four main processes in fingerprint recognition: Pre-processing, minutiae extraction, post processing and matching.

\section{Pre-Processing}

Pre-processing is an important step in fingerprint recognition. By implementing a good pre-processing technique, the minutiae can be extracted easily. In this step, fingerprint images are enhanced using Short Time Fourier Transform (STFT) (Chikkerur et al., 2007), which is based on contextual filtering in Fourier domain. After enhancing the image, binarization is implemented to transform the enhanced image into a binary image. It changes the pixel value into black for ridges and white for furrow. In addition, by improving the contrast between ridges and furrows. There are three main approaches in binarization (Meenen and Adhami, 2005): Global approach, neighbourhood-based approach and filter-based approach. In this study, local adaptive thresholding which based on neighbourhood-based approach is used because it has fast speed, good robustness and low complexity. Furthermore, the segmentation is implemented to eliminate the area near the boundary and area that does not have ridges.

The last process in pre-processing is thinning. Thinning is a process to change the pixel value into almost one (Xie and Lam, 1992). It reduces the thickness of the lines as much as possible with minimum loses. In thinning process, there are several processes, such as: (i) The output line should be a single pixel, (ii) the output line should not have any discontinuity, (iii) the output line should return to the centre pixel and (iv) eliminate the redundant line and unwanted pixels.

\section{Minutiae Extraction}

Crossing Number $(\mathrm{CN})$ technique is implemented for the minutiae extraction process. The minutiae are detected by analysing the nearby neighborhood of every ridge pixel in the image by utilizing $3 \times 3$ window. The $\mathrm{CN}$ value is defined as half of the aggregate of the differences between the pairs of adjacent pixels in the eight-neighbourhood. Below is the formula to calculate the Crossing Number value:

$$
C N=\frac{1}{2} \sum_{i=1}^{8}\left|P_{i}-P_{i-1}\right|, P_{9}=P_{1}
$$

where, $P_{i}$ is the pixel value in the $\mathrm{P}$ neighbourhood.

After detecting the $\mathrm{CN}$ value, the pixel can be classified based on its $\mathrm{CN}$ value. In this fingerprint system, only ridge ending and bifurcation are extracted. The ridge pattern is called ridge ending if Crossing Number value is one and it is bifurcation if the Crossing Number value is 3 .

Table 1. Comparison of Fusion levels

\begin{tabular}{lll}
\hline Level of Fusion & Strengths & Weaknesses \\
\hline Data Level & Richest data information & Contain the most noise Hard to get \\
Feature Level & Contain more information compared & Hard to implement when features \\
& with score and decision level & set size are not same \\
Score Level & Lesser noise compared with data level & Less information compared with \\
& Easy to implement & data and feature level \\
Lecision Level & Less noise & \\
& Eost popular method & Least information \\
\hline
\end{tabular}




\section{Post Processing}

Not all minutiae detected from fingerprint image are real minutiae. By implementing post processing, false minutiae can be reduced. There are several steps to eliminate the false minutiae: Short breaks removal, spurs removal, H-points removal, close minutiae removal and border minutiae removal (Zhao and Tang, 2007): False minutiae can exist due to dirt, oiliness and scanner issues.

\section{Matching}

Before matching the testing fingerprint with template fingerprint, alignment process needs to be implemented because the fingerprint position might be different. In addition, the robustness of the fingerprint recognition can be improved. In this study, Affine transformation is used to uniformly the distorted fingerprint image. Moreover, affine transformation transforms the variable into a new variable by using a combination of rotation, translation, scaling and shearing. Below is the formula to define the affine transformation.

In matching process, the minutiae features from the template are compared with the query minutiae features. In this stage, Modified Hausdorff Distance (MHD) is implemented. MHD is basically based on the Hausdorff Distance. Originally, this method has been proposed by (Dubuisson and Jain, 1994) for object matching and they determine the best distance measure based on the presence of noise. Compared to the original Hausdorff distance, MHD is more robust because by considering the average of the single distance, the effect of noise can be minimalized.

\section{Face Recognition}

Similar to fingerprint recognition, face images need to go through several processes before matching process. In this study, feature based face recognition is chosen as it is more robust compared with holistic based. In addition, a new method that combined Local Binary Pattern (LBP) and Scale Invariant Feature Transform (STFT) is introduced. This proposed method uses LBP to enhance the image before feature extraction is done by SIFT.

\section{Pre-processing}

The first step in pre-processing is face detection. In this study, face detection is done by using Haar featurebased cascade classifier which introduced by (Viola and Jones, 2001).

Next, the face image color is changed into grayscale image and LBP is performed to enhance the face image. In LBP method, if the neighbour pixel has a higher value than a center pixel, it will become one and if less than the center pixel, the pixel value will become 0 . After that, the value of the center pixel can be obtained by concatenating the eight neighbour pixel values. In this research, LBP is utilized to improve the image in light of its robustness and straightforwardness to monotonic gray-scale changes caused by illumination variations.

\section{Feature Extraction}

Scale Invariant Feature Transform (SIFT) is performed to extract the unique features from the enhanced image. This method is a feature based method and it is invariant to scale and rotation. There are four main steps in SIFT: Scale Space Extrema Detection which uses Difference of Gaussian, Keypoint localization which eliminates the weak keypoints, orientation assignment to make the keypoint invariance to rotation and keypoint descriptor.

\section{Matching}

After extracting the SIFT features, the matching process is performed. The best candidate match for each keypoints is obtained by finding its nearest neighbour in the keypoint database. In addition, the nearest neighbour is defined as the keypoint with the minimum Euclidian distance for the invariant descriptor vector. Furthermore, by comparing the closest neighbour distance with the second closest neighbour, the matching process can be more effective.

\section{Score Level Fusion}

Score level fusion is the fusion in matching score level. Therefore, it is additionally called matching level fusion (Cui and Yang, 2011; Jhansi and Reddy, 2015). In this research, the score level fusion is used to combined the matching score from fingerprint and face recognition. In addition, the extracted feature set of fingerprint and face are different so it is easier to fuse the information in score level. In this study, the score need to be normalized before fused together.

\section{Score Normalization}

In this study, Min-Max normalization is used for score normalization. Min-max normalization normalizes the scores to interval $[0,1]$. The normalized scores is calculated as follow:

$$
x^{\prime}=\frac{x-\min (X)}{\max (X)-\min (X)}
$$

where, $\min (X)$ and $\max (X)$ are the minimum and maximum values of the matching scores (Jain et al., 2005).

\section{Sum Rule}

Sum rule-based is performed to combined normalized fingerprint and face scores. The fused score is obtained by using the following formula: 


$$
f_{s}=x_{1}+\ldots+x_{m}
$$

In this research, weighted sum-rule is used. In weighted sum-rule, weight is added to the matching score so that the score can be set based on the weight. Below is the formula to calculate the weighted sum-rule:

$$
f_{s}=w_{1} x_{1}+\ldots+w_{m} x_{m}
$$

where, $w_{1}+w_{2}=1$. Moreover, $w_{i}$ is the weight which assigned to the normalized score $\left(x_{1}, \ldots, x_{m}\right)$.

\section{Experimental Results and Discussion}

\section{Dataset and Experiment Setting}

In this experiment, three fingerprint databases and two face databases were used. In addition, there are three combined databases that used to test the fusion performance. The detail of each database is explained in each subsection.

\section{Fingerprint Databases}

The fingerprint databases used in this experiment are FVC2002 DB1 database refers as FingerDB1 and FVC2002 DB2 database refer as FingerDB2. Each database consists of 100 person with 8 different fingerprint impression for each person. In total, there are 800 fingerprint images for each of database. FVC2002 DB1 database was collected using optical sensor "TouchView II" by Identix. Moreover, the image size is $388 \times 374$ with a resolution of $500 \mathrm{dpi}$. In addition, FVC2002 DB2 database was collected using optical sensor "FX2000" by Biometrika. The image size is $296 \times 560$ and the resolution is $569 \mathrm{dpi}$.

In addition, SDUMLA-HMT fingerprint database refers as FingerDB3 is used as well (Yilong et al., 2011). This database comprises of unique finger impression pictures from the index finger, middle finger and thumb finger of both hands. Furthermore, fingerprint images are captured by 5 different fingerprint sensor, such as AES2501 fingerprint scanner created by AuthentecInc, FPR620 optical finger impression scanner which created by ZhongzhengInc, URU4000 optical unique finger impression scanner created by ZhongkongInc and ZY202-B optical fingerprint scanner created by Chuba Academy of Sciences. There are 8 impressions captured for each of 6 fingers so the total images in the database are 106 subjects $8 \times 6 \times 5=25,440$ fingerprint images. Every fingerprint images in this database are saved in gray-level "bmp" format.

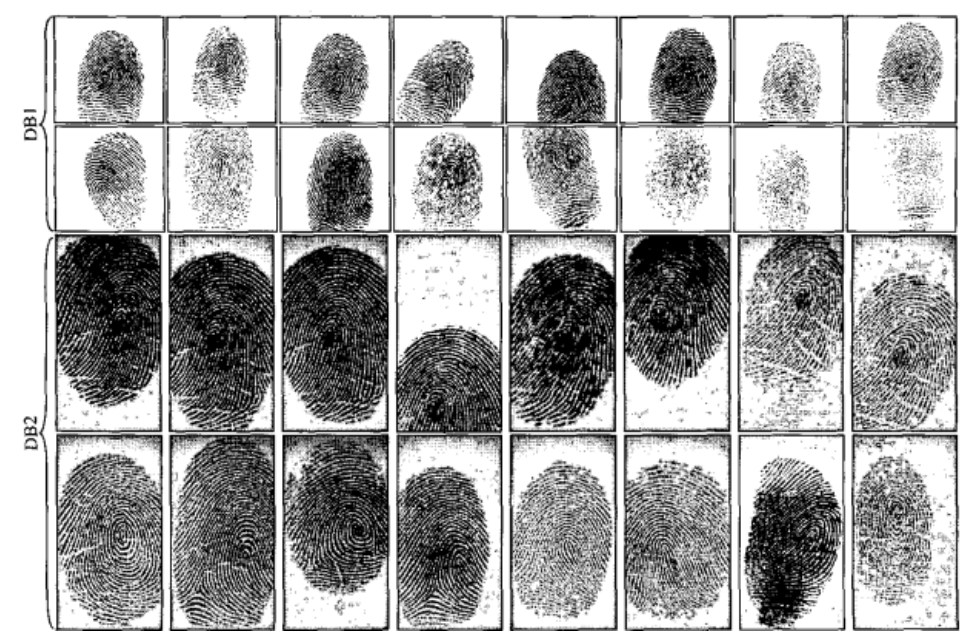

Fig. 1. FVC2002 DB1 and DB2 fingerprint database

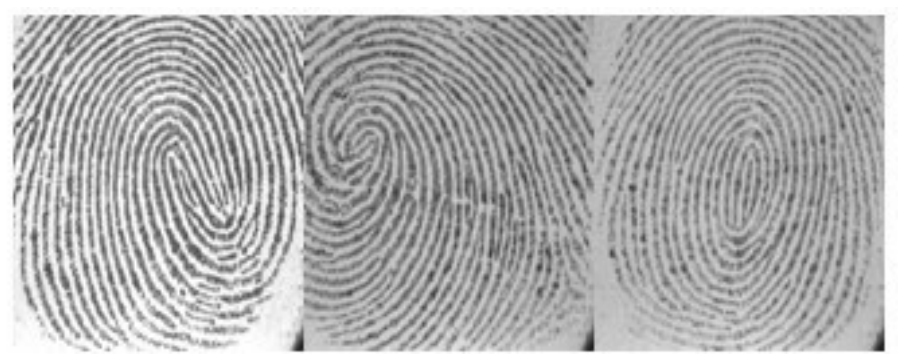

Fig. 2. SDUMLA-HMT fingerprint database 
In addition, Fig. 1 shows the fingerprint sample from FVC2002 DB1 and DB2 fingerprint database and Fig. 2 shows the fingerprint sample from SDUMLA-HMT Database.

\section{Face Databases}

There are two face databases that used for this experiment which is FERET database refer as FaceDB1 and SDUMLA-HMT refer as FaceDB2 (Yilong et al., 2011). These two databases were chosen because both of database consist of face images from more than 100 persons. The FERET database contains 1564 sets of images for a total of 14,126 images from 1199 persons and 365 duplicate sets of images (Delin et al., 2015). The Face Recognition Technology (FERET) program is conducted by the Defense Advance Research Project Agency (DARPA) and the National Institute of Standard and Technology (NIST). Figure 3 shows some sample images from FERET database.

Another database used in this experiment is SDUMLA-HMT face database. Figure 4 shows some sample images from SDUMLA-HMT database. This face database is collected with various poses, accessories, illumination and facial expression from 106 persons. Similar with FingerDB3, this database was created by Shendong University in 2010. In this study, only 800 face images from 100 persons are used. The original size of the image is $620 \times 480$ pixels. In this experiment, the detected face is cropped to $60 \times 90$ pixels.

\section{Fingerprint Matching using Modified Hausdorff} Distance

In this experiment, the fingerprint image was enhanced using STFT and the fingerprint feature was extracted using Crossing Number Technique. In addition, in point based matching, fingerprint alignment is very important as the finger position is different from one finger with another finger. In this study, an affine transformation is implemented. The affine transformation is used for rotation and translation purpose. For the rotation, the features are rotated from 0 until 360 degrees and translated from 0 until 10 point to find the closest match. In addition, MHD is used for fingerprint matching purpose.

Table 2 shows the difference between fingerprint matching using MHD with and without using alignment method on FingerDB1. As shown in Table 4, the EER with affine transformation is $18.55 \%$. Furthermore, on FingerDB2, the EER before alignment process is $39.64 \%$ and after implementing affine transformation, the EER can be reduced to $27.47 \%$. Lastly, the EER before alignment process on FingerDB3 is $28.79 \%$ and the EER can be reduced to $14.47 \%$ after implementing affine transformation.

Based on the experiment result, the alignment process especially affine transformation is very important in point based fingerprint matching. In addition, compared with other distance matching method, MHD is more robust as it uses the average of the single distance, the effect of noise can be minimised.

\section{Face Recognition using LBP-SIFT}

In this research, face features are extracted using three different methods: SIFT, LBP-SIFT and SURF. The intention of this experiment is to compare and verify that the proposed method has better result compared with the similar methods.

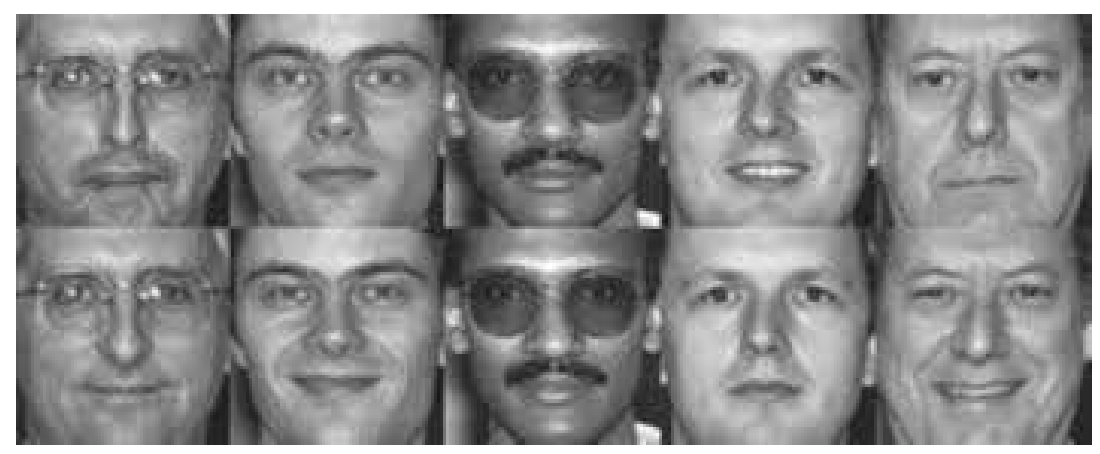

Fig. 3. FERET database
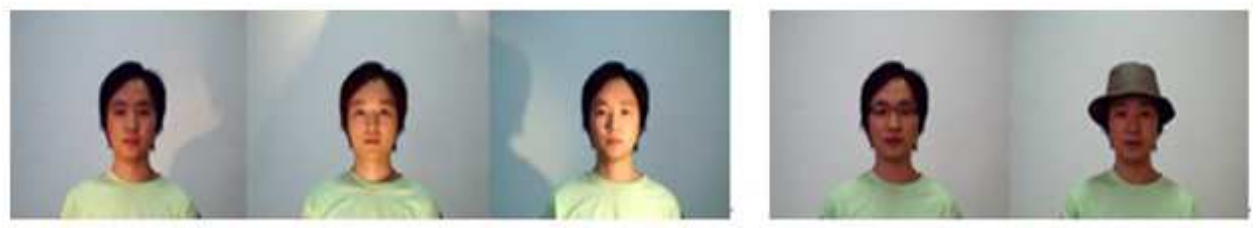

Fig. 4. SDUMLA-HMT face database 
Table 2. Performance comparison

\begin{tabular}{lll} 
& EER (\%) & \\
Database & MHD & MHD with affine transformation \\
\hline FingerDB1 & 30.38 & 18.55 \\
FingerDB2 & 39.64 & 27.47 \\
FingerDB3 & 28.79 & 14.47 \\
\hline
\end{tabular}

Table 3. Face recognition on FaceDB1

\begin{tabular}{ll}
\hline Method & EER \\
\hline SIFT & 16.4170 \\
LBP-SIFT & 10.8030 \\
SURF & 24.5695 \\
\hline
\end{tabular}

Table 4. Face recognition on FaceDB2

\begin{tabular}{ll}
\hline Method & EER \\
\hline SIFT & 13.930 \\
LBP-SIFT & 13.161 \\
SURF & 29.360 \\
\hline
\end{tabular}

Table 5. Score fusion

\begin{tabular}{ll}
\hline Databases & EER (\%) \\
\hline FaceDB1+FingerDB1 & 7.83 \\
FaceDB1+FingerDB2 & 9.13 \\
FaceDB2+FingerDB3 & 7.01 \\
\hline
\end{tabular}

Table 3 shows the face recognition method comparison on FaceDB1. SURF can achieve EER $24.569 \%$ and EER for original SIFT and LBP-SIFT are 16.417 and $24.5695 \%$ respectively.

In addition, Table 4 shows the EER comparison between SIFT, LBP-SIFT and SURF, on FaceDB2. Based on above table, SURF which is another point based method only can achieve EER 29.36\%. Meanwhile, LBP-SIFT can achieve EER 13.161\%. Based on Table 4 and 3, LBP-SIFT has a better performance compared with original SIFT and SURF.

\section{Fusion}

The score level fusion is used to combined the matching score from fingerprint and face recognition. Here, Sum rule-based analysis is performed to combined normalized fingerprint and face scores.

Table 5 shows that there are three scenarios that used in this experiment. The first database combination is FaceDB1 with FingerDB1, the second combination is FaceDB1 with FingerDB2 and the last is FaceDB2 with FingerDB3. The first two combinations consist of fingerprint and face images, not from the same person and the last combination is the database that collected from the same person. In addition, the EER score for fingerprint recognition is taken from MHD+affine transformation method and the EER score for face recognition is taken from LBP-SIFT method.

The first scenario can achieve EER $7.83 \%$, the second scenario is $9.13 \%$. The second scenario has a lower performance because FingerDB2 is more complex than FingerDB1. Lastly, the third scenario can achieve EER $7.01 \%$. These results are obtained after min-max normalization followed by the sum-rule method to combine the matching score.

\section{Conclusion}

In this research, we have presented a new approach for multimodal biometric using fingerprint and face recognition on score level fusion. From our findings, the fingerprint recognition, MHD along with the use of affine transformation can improve the accuracy performance. In addition, LBP-SIFT has shown better performance than original SIFT and SURF. Furthermore, by combining fingerprint and face recognition, the EER score can be greatly improved. Therefore, the experimental result shows that our proposed fusion method appears to be promising with $7.01 \sim 9.13 \%$ of EER based on different datasets.

\section{Author's Contributions}

All authors equally contributed in this work.

\section{Ethics}

This article is original and contains unpublished material. The corresponding author confirms that all of other authors have read and approved the manuscript and no ethical issues involved.

\section{References}

Chikkerur, S., N.C. Alexander and G. Venu, 2007. Fingerprint enhancement using STFT analysis. Patt. Recogn., 40: 198-211.

DOI: 10.1016/j.patcog.2006.05.036

Delin, C., L.Z. Liao, M.K.P. Ng and X. Wang, 2015. Incremental linear discriminant analysis: A fast algorithm and comparisons. IEEE Trans. Neural Netw. Learn. Syst., 29: 2716-2735.

DOI: 10.1109/TNNLS.2015.2391201

Dubuisson, M.P. and A.K. Jain, 1994. Modified Hausdorff distance for object matching. Proceedings of the 12th IAPR International Conference on Pattern Recognition, Oct. 9-13, IEEE Xplore Press, pp: 566-568. DOI: 10.1109/ICPR.1994.576361

Cui, F. and G. Yang, 2011. Score level fusion of fingerprint and finger vein recognition. J. Comput. Inform. Syst., 7: 5723-5731. 
Jain, A., K. Nandakumar and A. Ross, 2005. Score normalization in multimodal biometric systems. Patt. Recogn., 38: 2270-2285. DOI: 10.1016/j.patcog.2005.01.012

Jhansi, Y. and E.S. Reddy, 2015. A methodology for sketch based image retrieval based on score level fusion. Int. J. Comput. Applic., 109: 9-13. DOI: $10.5120 / 19167-0629$

Meenen, P. and R. Adhami, 2005. Approaches to image binarization in current automated fingerprint identification systems. Proceedings of the 37th Southeastern Symposium on System Theory, Mar. 20-22, IEEE Xplore Press, Tuskegee, Alabama, pp: 276-281.

DOI: $10.1109 / \mathrm{SSST} .2005 .1460921$

Ross, A. and A. Jain, 2003. Information fusion in biometrics. Patt. Recogn. Lett., 24: 2115-2125. DOI: $10.1016 / \mathrm{S} 0167-8655(03) 00079-5$

Ross, A. and A.K. Jain, 2004. Multimodal biometrics: An overview. Proceeding of the 12th European Signal Processing Conference, (SPC' 04), Vienna, pp: 1221-1224.
Sarhan, S., S. Alhassan and S. Elmougy, 2016. Multimodal biometric systems: A comparative study. Arab. J. Sci. Eng. DOI: $10.1007 / \mathrm{s} 13369-016-2241-0$

Viola, P. and M. Jones, 2001. Rapid object detection using a boosted cascade of simple features. Proceedings of the IEEE Computer Society Conference on Computer Vision and Pattern Recognition, Dec. 8-14, IEEE Xplore Press. DOI: 10.1109/CVPR.2001.990517

Xie, X. and K.M. Lam, 2006. An efficient illumination normalization method for face recognition. Patt. Recogn. Lett., 27: 609-617. DOI: 10.1016/j.patrec.2005.09.026

Yilong, Y., L. Liu and X. Sun, 2011. SDUMLA-HMT: A multimodal biometric database. Proceedings of the 6th Chinese Conference on Biometric Recognition, Dec. 3-4, Springer, Beijing, China, pp: 260-268. DOI: 10.1007/978-3-642-25449-9 33

Zhao, F. and X. Tang, 2007. Preprocessing and postprocessing for skeleton-based fingerprint minutiae extraction. Patt. Recogn., 40: 1270-1281. DOI: $10.1016 /$ j.patcog.2006.09.008 